\title{
Controversies in ocular tuberculosis
}

\author{
Marcus Ang, ${ }^{1,2,3}$ Soon-Phaik Chee ${ }^{1,2,3}$
}

${ }^{1}$ Singapore National Eye Centre, Singapore, Singapore

${ }^{2}$ Singapore Eye Research Institute, Singapore, Singapore

${ }^{3}$ Department of Ophthalmology and Visual Sciences, Duke-

National University of Singapore Graduate Medical School, Singapore, Singapore

\section{Correspondence to} Dr Marcus Ang, Singapore National Eye Centre, 11 Third Hospital Avenue, Singapore 168751, Singapore;

marcus.ang@snec.com.sg

Received 18 August 2016 Revised 12 October 2016 Accepted 18 October 2016 Published Online First 4 November 2016

\section{ABSTRACT}

Ocular tuberculosis still remains a presumptive, clinical diagnosis in the presence of supportive clinical signs and investigations, while in the absence of other possible causes of uveitis. The purpose of this review is to discuss three controversies of ocular tuberculosis today. First, it is unclear from many reports on how the diagnosis of ocular tuberculosis was defined, and if they included 'probable' or 'possible' diagnoses. Thus, there is a need to standardise the terminology used for ocular tuberculosis to allow for comparisons among studies. Second, the investigative approach is heterogeneous worldwide and there is currently no agreement on the pathogenesis of ocular tuberculosis. A suggested clinical approach involves first identifying supportive ocular signs, followed by a step-ladder approach of using various investigations such as nucleic acid amplification tests and interferon- $\gamma$ release assays, before confirming a 'definite' case of ocular tuberculosis. Third, there are currently no guidelines for the commencement or duration of antitubercular therapy in patients with ocular tuberculosis. The current review highlights the need for a collaboration from ophthalmologists around the world to establish a consensus on the terminology, guidelines on first-line investigations to use and guidance on antitubercular and corticosteroid therapy for ocular tuberculosis.

\section{INTRODUCTION}

In 1966, Duke-Elder opined that ocular tuberculosis (OTB) should be diagnosed in "cases of miliary or proliferative lesions in which there is no evidence of other disease liable to cause a granulomatous uveitis and which exhibit tuberculous disease elsewhere, particularly if specific therapy induces a favourable response'. ${ }^{1}$ Today, OTB in most cases still remains a presumptive, clinical diagnosis-not too far different from what was first described many years ago as we still do not have an ideal diagnostic test for OTB. In most cases, clinicians may only commit to 'probable' and 'possible' diagnoses of OTB in the presence of clinical signs and supportive investigations, ${ }^{2}$ in the absence of other possible causes of uveitis. ${ }^{3}$ The purpose of this review is to discuss the three main controversies of OTB today, with respect to the disease terminology, diagnostic challenges and dilemmas in management.

\section{CONTROVERSY NO. 1: TERMINOLOGY OF OTB}

The global burden of tuberculosis (TB) is still significant today-an estimated 9 million people with active $\mathrm{TB}$, and a third of the world's population with suspected Mycobacterium tuberculosis (MTB) infection. ${ }^{4}$ The WHO reported an increasing number of TB infections in both the low/ middle-income and high-income countries due to multidrug-resistant TB, HIV and global migration. ${ }^{5}$ This globalisation and resurgence of TB means that ophthalmologists now see a spectrum of disease: from eyes with obvious clinical OTB to mildly symptomatic patients with uveitis associated with an occult $\mathrm{TB}$ infection. ${ }^{5-7}$

However, the heterogeneous definition of OTB and the terminology of 'probable' and 'possible' OTB, which have resulted from the lack of an ideal specific test, may have contributed to the variability in reported prevalence of OTB. For example, the reported incidence of ocular involvement in patients with $\mathrm{TB}$ infection ranges from $1.4 \%$ in a sanatorium study by Donahue in 1967 to $16 \%$ in a highly endemic population in Saudi Arabia and $18 \%$ in patients with culture-proven TB in Spain. ${ }^{8}$ On the other hand, some studies report OTB as a percentage of all uveitis cases, which may range from $7 \%$ to $10.5 \%{ }^{8}$ Cross-sectional studies estimate a range from $1 \%$ to $4 \%$ areas with low $\mathrm{TB}$ endemicity, such as the USA, Europe and Japan, to $10 \%-26 \%$ in highly endemic regions such as India and Saudi Arabia. ${ }^{9}$ Even the reports of OTB prevalence within a country vary according to region, from $<1 \%$ in tertiary referral clinics to $10 \%$ or more in highly endemic regions of North America. ${ }^{2}$ In Europe, the percentage of patients with OTB from endemic countries ranges from $13.5 \%$ to $85 \% .^{10}$ However, it is unclear from many of these studies on how the diagnosis of OTB was defined and if they included 'probable' or 'possible' diagnoses. Thus, we are uncertain if the wide variability of OTB reported is true or a result of a lack of standardisation of the definition of the disease.

Therefore, there remains an important unmet need to standardise the terminology used for OTB diagnosis and a consensus among clinicians when reporting the prevalence of OTB-before we can truly appreciate the burden of this disease and compare study populations.

\section{CONTROVERSY NO. 2: DIAGNOSIS OF OTB}

The controversy in the diagnosis of OTB may stem from the uncertainty in the actual disease pathogeneses: Is this condition a TB infection of the eye or inflammation associated with a remote or systemic TB infection? ${ }^{11}$

To prove the first hypothesis, MTB has to be detected from within the eye. However, detection of acid-fast bacilli (AFB) with Ziehl-Neelsen or auramine-rhodamine stains has a low yield from the aqueous or vitreous. ${ }^{12}$ Cultures have an even lower yield, are laborious and may take $6-8$ weeks. ${ }^{13}$ It is yet more rare to obtain histopathologic evidence of necrotising granulomatous inflammation from an ocular biopsy, which would need to be sufficiently large to support the diagnosis of $\mathrm{OTB}^{14}{ }^{15}$ in the 
presence of AFB or supporting evidence of MTB DNA. ${ }^{16} 17$ The above techniques which attempt to isolate MTB from ocular samples may be challenged by the low sample volume and the paucibacillary nature of the disease. ${ }^{18}$ Alternatively, the poor positive yield of these results may be explained by the second hypothesis of OTB being an immune-mediated mechanism of inflammation. ${ }^{19}$ Nucleic acid amplification tests may be another method of detecting MTB DNA from the eye, but it lacks comparisons to culture as a gold standard ${ }^{20}$ and is unable to distinguish active infection from latent. Furthermore, these tests are affected by the volume of the sample, method of DNA extraction, number of targets used for amplification and presence of inhibitors in the sample. ${ }^{18}$ The specificities of these tests are high but sensitivities have been variable, that is, PCR: 33\%$67 \%,{ }^{21}$ quantitative real-time PCR: $57 \%,{ }^{22}$ multiplex PCR: $78 \%$ and loop-mediated isothermal amplification test: $86 \% .^{23}$ Moreover, all these tests usually require specialised and/or expensive laboratory equipment or reagents.

On the other hand, a patient presenting with uveitis and some evidence of pulmonary or systemic TB may not actually have OTB. ${ }^{10}$ The chest X-ray (CXR), which images the most common primary TB infection site, is normal in up to $70 \%$ of the patients with OTB. ${ }^{16}$ Radiological signs such as hilar lymphadenopathy and parenchymal scarring may not be specific of pulmonary TB. ${ }^{24}$ In cases where the CXR is inconclusive, more advanced radiological techniques such as CT or positron emission tomography scans may be useful to discern hilar, parenchymal or pleural disease. ${ }^{25-27}$ Immunological tests such as the tuberculin skin test (TST) lack specificity and do not distinguish latent from active TB disease, ${ }^{28}{ }^{29}$ with reported sensitivity: $71 \%$ (95\% CI $65 \%$ to $74 \%)$ and specificity $66 \%$ (95\% CI $46 \%$ to $86 \%) .{ }^{30}$ Newer blood tests such as the interferon- $\gamma$ release assays (IGRA) that include the QuantiFERON-Gold In-Tube (Cellestis, Carnegie, Australia) or T-SPOT.TB (Oxford Immunotec, Abingdon, UK) have gained popularity in recent years. ${ }^{31-34}$ The main advantage of IGRA is that it is an objective, reproducible blood test that requires only one visit. ${ }^{35}$ However, its disadvantages are the inability to distinguish latent from active infection, a higher cost, with time and temperaturesensitive sample processing. ${ }^{36}$ While evidence suggests that IGRA have better sensitivities and specificities compared with the TST, there is currently no consensus on its use (single vs combination with TST or CXR) with regard to the screening and diagnosis of TB. ${ }^{37-39}$ False-positive results are often a result of the low positive predictive value of the diagnostic test in areas that have very low pretest probability of disease. ${ }^{40}$ In other words, a negative test effectively rules out TB, but false positives are common; ${ }^{40}$ for example, in sarcoidosis where elevated interferon- $\gamma$ levels are seen in some patients with uveitis. ${ }^{41}$

Due to the difficulty in its diagnosis, the investigative approach to OTB is heterogeneous worldwide. ${ }^{42}$ For example, TST may be preferred over IGRA in low/middle-income countries, and CT chest may be more commonly ordered in high-income countries to detect subtle pulmonary involvement. ${ }^{42}$ Cost-effective analyses have suggested using IGRA in high-risk groups, the higher unit cost largely compensated by cost savings through more targeted performance of CXRs, follow-up and chemoprevention, ${ }^{43} 44$ especially in migrant populations and patients on haemodialysis and tumour necrosis factor alpha (TNF- $\alpha$ ) blockade. ${ }^{45-47}$ In our own study, we found that combination of an IGRA with a TST had the most costeffective model in the diagnosis of TB. ${ }^{48}$

While there is currently no consensus on the clinical approach to the diagnosis of OTB, most clinicians agree that careful ocular evaluation provides an important role. The identification of clinical signs such as broad-based synechiae, retinal vasculitis, multifocal choroiditis and serpiginoid choroiditis may be suggestive in some endemic regions, ${ }^{49} 50$ although not as useful in non-endemic regions. ${ }^{51} 52$ Nonetheless, the protean nature of OTB infection means that patients may present with a whole spectrum of clinical signs, that is, from granuloma, nodular scleritis and interstitial keratitis in the anterior segment to caseating granulomas in the ciliary body or choroidal tubercles in the posterior segment. ${ }^{53}$ A suggested clinical approach involves first identifying these suggestive clinical signs, followed by a step-ladder approach to confirm the diagnosis: (1) after the exclusion of other causes of uveitis, (2) the results of TST, IGRA, CXR or CT chest and (3) biopsy with molecular testing would be considered, before confirming a 'definite' case of OTB. ${ }^{54}$

\section{CONTROVERSY NO. 3: TREATMENT OF OTB}

There are currently no guidelines for the commencement or duration of anti-TB therapy (ATT) in patients with OTB. ${ }^{55}$ Whether OTB is a true infection of the eye or related to a systemic occult TB infection, at least it is known that ATT decreases the lifetime risk of developing active TB by $80 \%-90 \%$ in those with latent TB infections. ${ }^{30}$ Thus, it is believed that ATT could help eliminate the MTB inciting ocular inflammation either from within the eye or elsewhere in the body. ${ }^{56-59}$ Once the risks and benefits of initiating OTB have been weighed, the typical treatment regimen is to begin a combination of both systemic corticosteroids and ATT, typically a combination of isoniazid, rifampin, ethambutol and pyrazinamide. Some authors also recommend the consultation of an infectious disease specialist in the management, as many of these drugs may evoke allergic reactions or incur intolerable side effects. ${ }^{60}$

As there are currently no guidelines on ATT duration, most patients with OTB receive 6 months of the standard regimen. ${ }^{61}$ However, a recent survey among ophthalmologists revealed a large variability in the duration of ATT or when therapy is discontinued in non-responders. ${ }^{62}$ One study even suggested that a longer duration of more than 9 months ATT is required for the treatment of OTB. ${ }^{55}$ To reduce the risk of overtreating patients with uveitis and false-positive TB results, ${ }^{63}$ others have suggested raising the threshold of cut-off values such as that used in TST or IGRA before initiating ATT. ${ }^{64}$ It has also been recently suggested that some patients with higher IGRA responded with better treatment success. ${ }^{65}$

However, it is important to note that in many of these studies, the definition of clinical and treatment outcomes was inconsistent-largely due again to the lack of an exact definition of OTB, variation in duration and type of ATT and the variable use of concomitant corticosteroid therapy. ${ }^{65}$ Moreover, a lack of treatment response may be due to drug resistance, which is reported that up to $40 \%$ of patients with definite OTB, especially in patients originating from endemic countries. ${ }^{66}$ Therefore, a consensus is required to guide clinicians in the treatment and duration of OTB to avoid unnecessary treatment, overexposure to side effects and to the reduce the risk of introducing drug resistance. ${ }^{67}$

\section{CONCLUSION}

Recent advances in diagnostic tools for OTB such as molecular techniques for MTB DNA detection and immunological tests such as IGRA have improved the specificity of making this diagnosis. ${ }^{68}{ }^{69}$ However, the clinical diagnosis of OTB remains a complex issue, as these investigations are mainly adjunctive and 
complementary, while the clinical manifestations of OTB are protean. $^{53}$ The current review highlights the three main issues, which suggests that our approach to tackling this disease requires a collaboration from ophthalmologists around the world to establish (1) a consensus on the terminology and thus accurate reporting of OTB to allow comparisons among studies; (2) suggested guidelines on first-line investigations to use for endemic versus non-endemic regions and (3) guidance on treatment specifically for OTB in terms of systemic anti-TB and corticosteroid therapy.

Contributors All authors met the ICMJE (International Committee of Medical Journal Editors) criteria: substantial contributions to conception and design, acquisition of data or analysis and interpretation of data; drafting the article or revising it critically for important intellectual content and final approval of the version to be published.

\section{Competing interests None.}

Patient consent Obtained.

Provenance and peer review Not commissioned; externally peer reviewed.

Data sharing statement Additional unpublished data may be available from the corresponding author.

\section{REFERENCES}

1 Dinning WJ, Marston S. Cutaneous and ocular tuberculosis: a review. J R Soc Med 1985;78:576-81.

2 Cunningham ET, Jr., Rathinam SR, Albini TA, et al. Tuberculous uveitis. Ocul Immunol Inflamm 2015;23:2-6.

3 Gupta A, Sharma A, Bansal R, et al. Classification of intraocular tuberculosis. Ocul Immunol Inflamm 2015;23:7-13.

4 Conant MM, Vrasich CR, Wongskhaluang JV, et al. Role of the infectious disease consultant in management of patients with tuberculosis-associated ocular inflammation. Open Forum Infect Dis 2016:3:ofv195.

5 Global tuberculosis control: key findings from the December 2009 WHO report. Wkly Epidemiol Rec 2010;85:69-80.

6 Gandhi NR, Nunn P, Dheda K, et al. Multidrug-resistant and extensively drug-resistant tuberculosis: a threat to global control of tuberculosis. Lancet 2010;375:1830-43.

7 Blumberg HM, Migliori GB, Ponomarenko 0, et al. Tuberculosis on the move. Lancet 2010;375:2127-9.

8 Vos AG, Wassenberg MW, de Hoog J, et al. Diagnosis and treatment of tuberculous uveitis in a low endemic setting. Int J Infect Dis 2013;17:e993-9.

9 Abu El-Asrar AM, Abouammoh M, Al-Mezaine HS. Tuberculous uveitis. Int Ophthalmol Clin 2010;50:19-39.

10 Caspers L, Makhoul D, Ebraert H, et al. Clinical manifestations of patients with intraocular inflammation and positive QuantiFERON-TB gold in-tube test in a country nonendemic for tuberculosis. Am J Ophthalmol 2014;158:646-7.

11 Garip A, Diedrichs-Möhring M, Thurau SR, et al. Uveitis in a patient treated with Bacille-Calmette-Guerin: possible antigenic mimicry of mycobacterial and retinal antigens. Ophthalmology 2009;116:2457-62.e1-2.

12 Gupta V, Gupta A, Rao NA. Intraocular tuberculosis-an update. Surv Ophthalmol 2007;52:561-87.

13 Dinnes J, Deeks J, Kunst $H$, et al. A systematic review of rapid diagnostic tests for the detection of tuberculosis infection. Health Technol Assess 2007;11:1-196.

14 Wroblewski KJ, Hidayat AA, Neafie RC, et al. Ocular tuberculosis: a clinicopathologic and molecular study. Ophthalmology 2011;118:772-7.

15 Biswas J, Madhavan HN, Gopal L, et al. Intraocular tuberculosis. Clinicopathologic study of five cases. Retina 1995;15:461-8.

16 Sarvananthan N, Wiselka M, Bibby K. Intraocular tuberculosis without detectable systemic infection. Arch Ophthalmol 1998;116:1386-8.

17 Park DY, Kim JY, Choi KU, et al. Comparison of polymerase chain reaction with histopathologic features for diagnosis of tuberculosis in formalin-fixed, paraffin-embedded histologic specimens. Arch Pathol Lab Med 2003;127:326-30.

18 Sharma K, Gupta V, Bansal R, et al. Novel multi-targeted polymerase chain reaction for diagnosis of presumed tubercular uveitis. J Ophthalmic Inflamm Infect 2013;3:25

19 Ang $M$, Cheung G, Vania $M$, et al. Aqueous cytokine and chemokine analysis in uveitis associated with tuberculosis. Mol Vis 2012;18:565-73.

20 Parashar D, Chauhan DS, Sharma VD, et al. Applications of real-time PCR technology to mycobacterial research. Indian J Med Res 2006;124:385-98.

21 Arora SK, Gupta V, Gupta A, et al. Diagnostic efficacy of polymerase chain reaction in granulomatous uveitis. Tuber Lung Dis 1999;79:229-33.

22 Singh $\mathrm{R}$, Toor $\mathrm{P}$, Parchand $\mathrm{S}$, et al. Quantitative polymerase chain reaction for Mycobacterium tuberculosis in so-called Eales' disease. Ocul Immunol Inflamm 2012;20:153-7.
23 Balne PK, Barik MR, Sharma S, et al. Development of a loop-mediated isothermal amplification assay targeting the mpb64 gene for diagnosis of intraocular tuberculosis. J Clin Microbiol 2013;51:3839-40.

24 Joshi $\mathrm{R}$, Patil S, Kalantri S, et al. Prevalence of abnormal radiological findings in health care workers with latent tuberculosis infection and correlations with $T$ cell immune response. PLOS ONE 2007;2:e805.

25 Ganesh SK, Roopleen, Biswas J, et al. Role of high-resolution computerized tomography (HRCT) of the chest in granulomatous uveitis: a tertiary uveitis clinic experience from India. Ocul Immunol Inflamm 2011;19:51-7.

26 Mehta S. Patterns of systemic uptake of 18-FDG with positron emission tomography/computed tomography (PET/CT) studies in patients with presumed ocular tuberculosis. Ocul Immunol Inflamm 2012;20:434-7.

27 Doycheva D, Deuter C, Hetzel J, et al. The use of positron emission tomography/CT in the diagnosis of tuberculosis-associated uveitis. Br J Ophthalmol 2011;95:1290-4.

28 Albini TA, Karakousis PC, Rao NA. Interferon-gamma release assays in the diagnosis of tuberculous uveitis. Am J Ophthalmol 2008;146:486-8.

29 Ang M, Wong WL, Kiew SY, et al. Prospective head-to-head study comparing 2 commercial interferon gamma release assays for the diagnosis of tuberculous uveitis. Am J Ophthalmol 2014;157:1306-14; 14 e1-4.

30 Targeted tuberculin testing and treatment of latent tuberculosis infection. American Thoracic Society. MMWR Recomm Rep 2000;49:1-51.

31 Andersen P, Munk ME, Pollock JM, et al. Specific immune-based diagnosis of tuberculosis. Lancet 2000;356:1099-104.

32 Heydari H, Mutha NV, Mahmud MI, et al. StaphyloBase: a specialized genomic resource for the staphylococcal research community. Database (Oxford) 2014;2014: bau010.

33 Yesuratnam A, Wiesenfeld D, Tsui A, et al. Preoperative evaluation of oral tongue squamous cell carcinoma with intraoral ultrasound and magnetic resonance imaging-comparison with histopathological tumour thickness and accuracy in guiding patient management. Int J Oral Maxillofac Surg 2014;43:787-94.

34 Ang M, Htoon HM, Chee SP. Diagnosis of tuberculous uveitis: clinical application of an interferon-gamma release assay. Ophthalmology 2009;116:1391-6.

35 Kleinert S, Kurzai 0, Elias J, et al. Comparison of two interferon-gamma release assays and tuberculin skin test for detecting latent tuberculosis in patients with immune-mediated inflammatory diseases. Ann Rheum Dis 2010;69:782-4.

36 Ang $M$, Wong $W$, Ngan CC, et al. Interferon-gamma release assay as a diagnostic test for tuberculosis-associated uveitis. Eye (Lond) 2012;26:658-65.

37 Denkinger CM, Dheda K, Pai M. Guidelines on interferon-gamma release assays for tuberculosis infection: concordance, discordance or confusion? Clin Microbiol Infect 2011;17:806-14.

38 Kardos M, Kimball AB. Time for a change? Updated guidelines using interferon gamma release assays for detection of latent tuberculosis infection in the office setting. J Am Acad Dermatol 2012;66:148-52.

39 Ang $M$, Wong WL, Li $X$, et al. Interferon gamma release assay for the diagnosis of uveitis associated with tuberculosis: a Bayesian evaluation in the absence of a gold standard. Br J Ophthalmol 2013;97:1062-7.

40 Pepple KL, Van Gelder R, Forooghian F. Caveats about QuantiFERON-TB gold in-tube testing for uveitis. Am J Ophthalmol 2014;157:752-3.

41 La Distia Nora R, van Velthoven ME, Ten Dam-van Loon NH, et al. Clinical manifestations of patients with intraocular inflammation and positive QuantiFERON-TB gold in-tube test in a country nonendemic for tuberculosis. Am J Ophthalmol 2014;157:754-61.

42 Lou SM, Montgomery PA, Larkin KL, et al. Diagnosis and treatment for ocular tuberculosis among uveitis specialists: the international perspective. Ocul Immunol Inflamm 2015;23:32-9.

43 Hardy AB, Varma R, Collyns T, et al. Cost-effectiveness of the NICE guidelines for screening for latent tuberculosis infection: the QuantiFERON-TB Gold IGRA alone is more cost-effective for immigrants from high burden countries. Thorax 2010:65:178-80

44 Nienhaus A, Schablon A, Costa JT, et al. Systematic review of cost and costeffectiveness of different TB-screening strategies. BMC Health Serv Res 2011;11:247.

45 Pareek M, Baussano I, Abubakar I, et al. Evaluation of immigrant tuberculosis screening in industrialized countries. Emerging Infect Dis 2012;18:1422-9.

46 Pareek M, Bond M, Shorey J, et al. Community-based evaluation of immigrant tuberculosis screening using interferon gamma release assays and tuberculin skin testing: observational study and economic analysis. Thorax 2013;68:230-9.

47 Kowada A. Cost effectiveness of the interferon-gamma release assay for tuberculosis screening of hemodialysis patients. Nephrol Dial Transplant 2013;28:682-8.

48 Ang M, Nguyen HV, Kiew SY, et al. Cost-effectiveness of alternative strategies for interferon-gamma release assays and tuberculin skin test in tuberculous uveitis. Br J Ophthalmol 2015;99:984-9.

49 Gupta A, Bansal R, Gupta V, et al. Ocular signs predictive of tubercular uveitis. Am J Ophthalmol 2010;149:562-70.

50 Ang M, Hedayatfar $A$, Zhang $R$, et al. Clinical signs of uveitis associated with latent tuberculosis. Clin Experiment Ophthalmol 2012;40:689-96.

51 Varma D, Anand S, Reddy AR, et al. Tuberculosis: an under-diagnosed aetiological agent in uveitis with an effective treatment. Eye (Lond) 2006;20:1068-73. 
52 Tognon MS, Fiscon M, Mirabelli P, et al. Tuberculosis of the eye in Italy: a forgotten extrapulmonary localization. Infection 2014;42:335-42.

53 Gupta V, Shoughy SS, Mahajan S, et al. Clinics of ocular tuberculosis. Ocul Immunol Inflamm 2015:23:14-24.

54 Ang M, Vasconcelos-Santos DV, Sharma K, et al. Diagnosis of ocular tuberculosis. Ocul Immunol Inflamm Published Online First: 5 Jul 2016. doi:10.1080/09273948. 2016.1178304

55 Ang $M$, Hedayatfar $A$, Wong $W$, et al. Duration of anti-tubercular therapy in uveitis associated with latent tuberculosis: a case-control study. Br J Ophthalmol 2012;96:332-6.

56 Bansal R, Gupta A, Gupta V, et al. Role of anti-tubercular therapy in uveitis with latent/manifest tuberculosis. Am J Ophthalmol 2008;146:772-9.

57 Tabbara KF. Tuberculosis. Curr Opin Ophthalmol 2007;18:493-501.

58 Morimura Y, Okada AA, Kawahara S, et al. Tuberculin skin testing in uveitis patients and treatment of presumed intraocular tuberculosis in Japan. Ophthalmology 2002;109:851-7.

59 Alvarez GG, Roth VR, Hodge W. Ocular tuberculosis: diagnostic and treatment challenges. Int I Infect Dis 2009;13:432-5.

60 Bodaghi B, LeHoang P. Ocular tuberculosis. Curr Opin Ophthalmol 2000;11: 443-8.

61 American Thoracic Society, CDC, Infectious Diseases Society of America. Treatment of tuberculosis. MMWR Recomm Rep 2003;52:1-77.
62 Lou SM, Larkin KL, Winthrop K, et al. Lack of consensus in the diagnosis and treatment for ocular tuberculosis among uveitis specialists. Ocul Immunol Inflamm 2015;23:25-31.

63 Mancuso JD, Mazurek GH, Tribble D, et al. Discordance among commercially available diagnostics for latent tuberculosis infection. Am J Respir Crit Care Med 2012;185:427-34.

64 Gineys R, Bodaghi B, Carcelain G, et al. QuantiFERON-TB gold cut-off value: implications for the management of tuberculosis-related ocular inflammation. Am J Ophthalmol 2011;152:433-40. e1.

65 Agrawal R, Gonzalez-Lopez JJ, Nobre-Cardoso J, et al. Predictive factors for treatment failure in patients with presumed ocular tuberculosis in an area of low endemic prevalence. Br J Ophthalmol 2016;100:348-55.

66 Fattorini L, Mustazzolu A, Piccaro G, et al. Drug-resistant tuberculosis among foreign-born persons in Italy. Eur Respir J 2012;40:497-500.

67 Forget EJ, Menzies D. Adverse reactions to first-line antituberculosis drugs. Expert Opin Drug Saf 2006;5:231-49.

68 Pai M, Zwerling A, Menzies D. Systematic review: T-cell-based assays for the diagnosis of latent tuberculosis infection: an update. Ann Intern Med 2008; 149:177-84.

69 Bansal R, Sharma K, Gupta A, et al. Detection of Mycobacterium tuberculosis genome in vitreous fluid of eyes with multifocal serpiginoid choroiditis. Ophthalmology 2015;122:840-50. 